Ann. Génét. Sél. anim., I974, 6 (I), I7-28.

\title{
CONSOMMATION ALIMENTAIRE D'ANIMAUX A PLUMAGE RÉDUIT (GENE Na) OU NORMAL EN PRÉSENCE OU EN L'ABSENCE DU GÈNE DE NANISME $d w$
}

\author{
P. MÉRAT et A. BORDAS \\ Laboratoire de Génétique factorielle, \\ Centre national de Recherches zootechniques, I. N. R. A., \\ 78350 Jouy en Josas
}

RÉSUMÉ

La consommation alimentaire individuelle observée, ou corrigée pour le poids corporel, le gain de poids et la production d'œufs, a été comparée pour des poules " cou nu " ou à plumage normal, en présence ou en l'absence du gène de nanisme $d w$ : l'excès de consommation "corrigée " des poules " cou nu " dans nos conditions de température (autour de $18-20^{\circ} \mathrm{C}$ en moyenne) est significatif. Chez les coqs, la différence est plus considérable, en pourcentage, que chez les poules. Elle est du même ordre chez les nains et chez les normaux, alors que chez les 웅, nos données suggèrent qu'elle pourrait être diminuée en présence du gène $d w$.

\section{INTRODUC'TION}

Le gène recessif lié au sexe $d w$ (HuTT, I953 et I959; BERNIER et ARSCOTT, I960) réduit le poids corporel d'environ $40 \mathrm{p}$. Ioo chez les coqs et $30 \mathrm{p}$. Ioo chez les poules. Apparu dans le troupeau expérimental de Jouy en Josas, il y est étudié en particulier pour son effet sur la consommation alimentaire des pondeuses.

Le gène " cou nu " $(N a)$, autosomal dominant, est caractérisé par une absence de plumes sur le cou et dans certaines autres zônes du corps, et par une réduction du sousplumage (HUTT, I949). Des résultats concernant la croissance et la production d'œufs (MÉRAT, données non publiées) laissent supposer qu'il ne serait pas avantageux d'introduire le gène dans une population de taille normale, dans nos conditions climatiques du moins, mais qu'il pourrait améliorer légèrement le poids des œufs et peut-être leur nombre en présence de gène de nanisme $d w$. 
A la suite de ces résultats, il était intéressant de connaître la consommation alimentaire des poules à " cou nu " ou à plumage normal en présence du gène $D w$ (taille normale) ou $d w$ (taille réduite).

Sur des jeunes de I9 à 55 jours et en présence de $d w$, Srmon (1972) ne note pas de différence significative de gain de poids, ni de consommation alimentaire, associée au locus $N a$. D'autre part, des interactions au niveau physiologique ont été trouvées, chez le poulet, entre les gènes $N a$ et $D w$ (TouchBurn et BLum, 1972).

Sur des coqs ou des poules adultes, on s'attend a priori à ce que les oiseaux à " cou nu ", ayant des déperditions énergétiques accrues, au moins en deçà de leur zone de neutralité thermique, aient une consommation d'aliment légèrement supérieure aux poules à plumage normal. Cependant, ceci n'a pas, à notre connaissance, été vérifié, et l'on n'a pas non plus cherché l'influence possible de la taille, et en particulier du gène $d w$, sur les effets associés au gène $N a$ à cet égard. Ceci est 1'objet du présent article.

\section{MATÉRIEI,}

Le présent travail a été répété trois années : En I971, sur une courte période, puis en 1972 et en 1973 .

Les animaux utilisés étaient issus du troupeau de Jouy en Josas, où divers gènes connus sont maintenus en ségrégation, notamment aux loci $D w$ et $\mathrm{Na}$. Comme nous l'avons indiqué plus haut, le gène $d w$ a été identifié dans cette population en I959; quant au gène $N a$, il y a été introduit en 1963 .

Chacune des trois années, les animaux expérimentaux provenaient de croisements où le père était hétérozygote $D w d w$ et les mères naines $(d w)$. L'un des deux parents était également hétérozygote pour $\mathrm{Na}(\mathrm{Na} n a)$ et l'autre homozygote récessif ( $n a n a)$. De cette façon, les différents génotypes comparés se retrouvaient à l'intérieur de chaque famille de frères-sœurs ou au moins de demi-frères et demi-sœurs. Dans les plus nombreuses de ces familles, des quadruplets d'individus des quatre génotypes possibles, par sexe, et si possible de poids relativement voisin, étaient retenus au départ de chaque expérience. En I97I, seules des femelles étaient utilisées. En 1972 et 1973, l'expérimentation portait d'une part sur des poules en ponte, de l'autre sur des coqs, issus des mêmes pères (et si possible des mêmes mères). Le tableau I indique, par année et par sexe, les effectifs et le nombre de familles de même père dont provenait chaque série. Seuls étaient utilisés les animaux dont toutes les performances étaient enregistrées pour l'ensemble de la période expérimentale à laquelle ils appartenaient.

TABLEAU I

Effectifs par génotype et nombres de familles de même père pour chaque série expérimentale

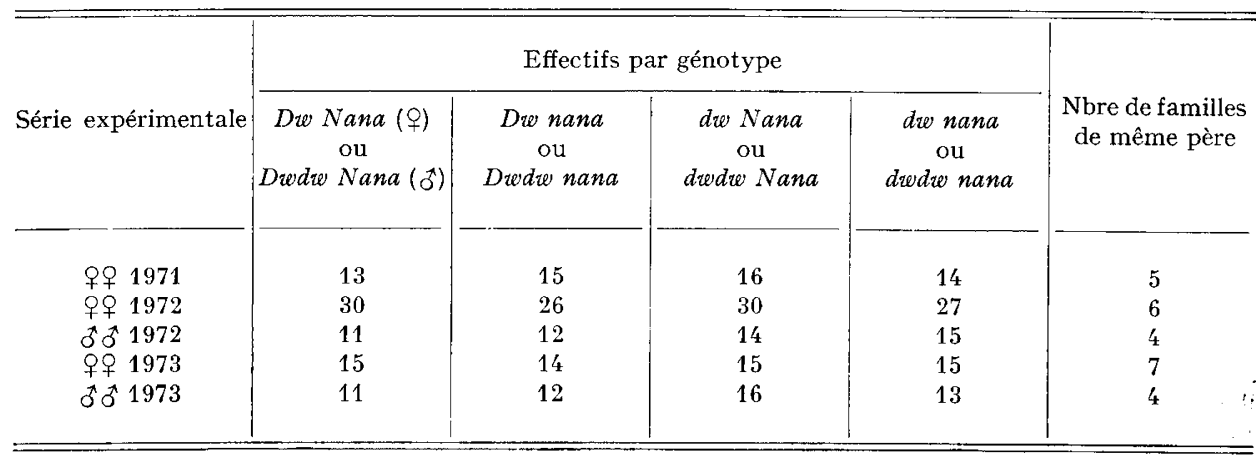


L'élevage des jeunes, éclos à l'automne, avait lieu au sol jusqu'à l'âge de I6 semaines, puis les animaux étaient mis en cages individuelles. Le contrôle individuel de la consommation alimentaire était fait au ro mois d'âge pendant seulement 17 jours pour les $\$ q$ de I971; en 1972 et 1973 dans chaque sexe ce contrôle commençait à 8 mois d'âge environ, et s'étendait sur 3 périodes de 28 jours, d'avril à juillet. A la fin de chaque période, chaque individu était pesé, ainsi que la quantité totale d'aliment ingérée, et le total des œufs produits pour les pondeuses ; une estimation individuelle était faite pour le gaspillage d'aliment, peu important dans nos conditions. En outre, au cours des deux années 1972 et 1973 , pendant deux semaines à la fin de la dernière période, on relevait la consommation journalière individuelle d'aliment et d'eau de boisson ; les données correspondantes seront présentées dans un autre article.

L'aliment était donné sous forme de farine. Il correspondait pour les deux sexes à une formule "reproducteurs " à I 6 p. I oo de protéines et environ $2520 \mathrm{kcal}$ d'énergie métabolisable $/ \mathrm{kg}$. Le même aliment était utilisé les trois années.

Le local où le contrôle de consommation individuelle avait lieu subissait partiellement les fluctuations de la température ambiante : Un chauffage d'appoint permettait de ne pas descendre en-dessous de $\mathrm{I}^{\circ} \mathrm{C}$ environ. Par ailleurs, les maxima journaliers en fin de période expérimentale (juin) pouvaient dépasser $25^{\circ} \mathrm{C}$ et même $30^{\circ}$ en de rares occasions. La température moyennc se situait dans l'ensemble au voisinage de ${ }^{\circ}{ }^{\circ} \mathrm{C}$ en 1972 et de $20^{\circ} \mathrm{C}$ en 1973.

\section{MÉTHODES}

Les variables enregistrées par animal et par période sont :

- le poids corporel, moyenne des poids en début et en fin de période ;

- le poids total des œufs pondus (pour les 웅) ;

- la consommation d'aliment;

- Ia variation de poids, obtenue par différence entre le poids enregistré à la fin et au début d'une même période.

Nous ne présentons ici que les résultats concernant, pour chaque variable, la moyenne des trois périodes (pour I972 et I973).

A partir de ces données, la consommation " théorique " $\mathrm{T}$ pour un individu est estimée en fonction de son poids moyen $P$, de sa variation de poids moyenne $\Delta P$ et, pour les poules, du poids des œufs pondus $\mathrm{E}$, suivant une équation de régression multiple de la forme :

$$
\text { ou } \begin{aligned}
\mathrm{T} & =a \mathrm{P}^{\alpha}+b \Delta \mathrm{P}+c \mathrm{E}+\mathrm{R} \text { (pour les } q \text { ) } \\
\mathrm{T} & \left.=a \mathrm{P}^{\alpha}+b \Delta \mathrm{P}+\mathrm{R} \text { (pour les ơ } \sigma^{\alpha}\right)
\end{aligned}
$$

Cette équation a été définie par BYERLy (I94I). R y représente un terme résiduel.

PRoD' номмe (1965) a déterminé pour nos conditions la valeur la meilleure de l'exposant $\alpha$ suivant le principe exposé par BYERLY. La méthode par approximations indique que l'on peut utiliser pour $\alpha$ une valeur voisine de 0,5 : nous avons donc adopté pour variable non le poids corporel mais sa racine carrée. A chaque expérience, les coefficients de régression de la variable dépendante sur chaque variable indépendante ont d'abord été estimés séparément pour chaque génotype (aux loci $D w$ et $N a$ ) ; après avoir vérifié que le génotype au locus $N a$ ne causait pas de différence significative pour ces coefficients, une équation unique a été retenue, d'une part pour l'ensemble des animaux de taille normale du même sexe chaque année, de l'autre pour l'ensemble des nains.

C'est à partir de cette équation commune que la consommation théorique $\mathrm{T}$ de chaque individu est estimée. Par différence avec sa consommation observée $O$, on en tire sa consommation "corrigée " : $\mathrm{R}=\mathrm{O}-\mathrm{T}$.

Nous considérons également une consommation individuelle " partiellement corrigée " $R$ ", différence entre la consommation observée et une consommation théorique non corrigée pour la variation de poids $\Delta \mathrm{P}$, c'est-à-dire obtenue à partir d'une équation de regression semblable à celle indiquée ci-dessus, mais où la variable indépendante $\Delta \mathrm{P}$ est omise.

Pour chaque variable $\left(\mathrm{P}, \Delta \mathrm{P}, \mathrm{E}, \mathrm{O}, \mathrm{R}, \mathrm{R}^{\prime}\right)$, une analyse de variance est faite sur l'ensemble des deux années I972 et I973 (les données de I97I sur les 우, correspondant à une courte durée, ne sont pas comparables aux autres et peuvent être considérées comme un essai préliminaire).

L'analyse est faite séparément par sexe et à l'intérieur de chacun des génotypes $D w$ et $d w$ (pour les + 우) ou $D w d w$ et $d w d w$ (pour les $\delta^{\star} \delta^{\star}$ ). Le fait de considérer à part ces génotypes est justifié en partie par la variance sensiblement inégale chez les animaux nains et chez les normaux nur toutes les variables considérées. D'autre part et surtout, la différence entre ces deux groupes, tant en ce qui concerne le poids corporel que la ponte et la consommation alimentaire, est déjà 
bien connue, de sorte que nous nous attachons davantage ici à estimer l'effet des génotypes au locus $N a$, chez des oiseaux de taille normale ou chez des nains.

De même, entre sexes, les variances du poids et des consommations observées ou corrigées sont nettement différentes, ce qui rend préférable une analyse séparée.

L'analyse de variance dans chaque lot expérimental et pour chaque variable tient compte des effectifs inégaux dans les sous-groupes (KENDALL et STUART, 1967). Elle comporte trois sources contrôlées de variation, le génotype au locus $N a$, l'année, et, à l'intérieur des années, la famille (de même père). La première de ces sources de variations a été considérée, en l'absence d'interaction significative avec les autres, comme un effet fixé, ces dernières correspondant à des effets aléatoires (SNEDECOR et COCHRAN, I967). Nous avons vérifié que, chez les femelles, la variance interaction génotype $\times$ année n'était nulle part significative. Quant à l'interaction génotype $\times$ famille sur l'ensemble des deux années, elle ne l'est pas non plus sauf pour la consommation observée des poules $D w$. Chez les coqs, de même, il n'apparaît nulle part d'interaction génotype $\times$ année significative, et la variance interaction génotype $\times$ famille ne l'est pas pour le poids et la variation de poids chez les normaux et chez les nains, et pour la consommation observée et corrigée chez les nains. Dans tous ces cas, nous avons testé l'effet du génotype au locus $N a$ par comparaison avec la variance résiduelle ; dans les cas restants, le dénominateur du rapport $\mathrm{F}$ considéré est la variance interaction génotype $\times$ famille. Par ailleurs, la variance "année " est testée par rapport à la variance "familles intra-années " lorsque cette dernière est significative.

En complément, et malgré les différences de variance déjà notées entre génotype nain et normal, nous avons fait pour la consommation observée, où l'effet de $d w$ était le plus considérable, une analyse de variance pour chaque sexe incluant les facteurs contrôlés "génotype au locus $D w$ ", "génotype au locus $\mathrm{Na}$ " et " année ", pour tester en particulier l'interaction possible entre les deux premiers facteurs.

Les variances "interaction " étaient estimées suivant la méthode approchée donnée par SNEDECOR et CoCHRAN ( 1967 ) pour le cas d'effectifs inégaux.

\section{RÉSUTTATS}

Les tableaux 2 et 3 contiennent les valeurs moyennes des performances observées respectivement chez les 우 et chez les $\hat{\sigma} \hat{\sigma}$ pour la durée totale de chaque série expérimentale par génotype. A côté de ces valeurs, pour les variables de consommation, est indiquée entre parenthèses la différence entre la moyenne des animaux à " cou nu " et celle des animaux na na, en p. roo de la consommation moyenne de ces derniers, parmi les oiseaux de taille normale d'une part, les nains de l'autre.

L'effet du gène dw dans les deux sexes, sur le poids corporel et la consommation alimentaire observée, apparait clairement et confirme des faits bien connus. Quant au gain de poids, on peut remarquer qu'il est plus faible, les deux années, pour les poules naines que pour les normales : ce fait est analysé séparément (MÉRAT et RICARD, I974).

Chez les poules, l'effet du gène " cou nu " est sensible pour la consommation observée, et plus encore pour la consommation corrigée totalement ou partiellement, et il est de même sens les trois années pour les 우워 de taille normale : Parmi celles-ci, les " cou nu » consomment plus d'aliment (de 3 à plus de 7 p. Ioo suivant l'année) que les poules complètement emplumées, à poids, gain de poids et ponte identiques. L'effet est de même sens, dans l'ensemble, chez les naines, mais il est à première vue plus faible.

Chez les coqs, le gène $N a$ augmente la consommation d'aliment dans une proportion encore plus grande que chez les $ㅇ ㅜ, q u$ 'il s'agisse de la consommation « observée " ou " corrigée ", et ce, de façon à peu près égale chez les nains ou chez les $\sigma^{\star} \delta$ de taille normale (I2 à I5 p. Ioo de plus selon le lot expérimental).

On peut rappeler en passant que les coefficients de l'équation de BYERLY, dans 
TABLEAU 2

Valeurs moyennes des performances par génotype (우우)

\begin{tabular}{|c|c|c|c|c|c|c|}
\hline $\begin{array}{l}\text { Année et } \\
\text { génotype }\end{array}$ & $\begin{array}{l}\text { Alim. consom. } \\
(\mathrm{g} / 28 \mathrm{j} \text { ou } \\
\mathrm{g} / 17 \mathrm{j} \text { en } 1971)\end{array}$ & $\begin{array}{c}\text { Consommation } \\
\text { corrigée }\end{array}$ & $\begin{array}{c}\text { Consommation } \\
\text { partiellement } \\
\text { corrigée }\end{array}$ & $\begin{array}{l}\text { Poids } \\
\text { corporel } \\
\text { (g) }\end{array}$ & $\begin{array}{l}\text { Variat. pds } \\
\text { ( } \mathrm{g} / 28 \mathrm{j} \text { ou } \\
\mathrm{g} / 17 \mathrm{j} \\
\text { en } 1971)\end{array}$ & $\begin{array}{l}\text { Pds œufs } \\
\text { pondus } \\
\text { g/28 j ou } \\
\text { g/17 j } \\
\text { en } 1971 \text { ) }\end{array}$ \\
\hline 1971 & & & & & & \\
\hline $\begin{array}{l}\text { Dw Nana } \\
\text { Dw nana } \\
\text { dw Nana } \\
\text { dw nana }\end{array}$ & $\begin{array}{l}2073(+6,1 \%) \\
1950 \\
1326(+1,3 \%) \\
1309\end{array}$ & $\begin{array}{l}+65(+5,0 \%) \\
-\quad 36 \\
+\quad 9(+3,3 \%) \\
-\quad 34\end{array}$ & $\begin{array}{l}+23(+3,2 \%) \\
-42 \\
-\quad 35(-2,2 \%) \\
-\quad 7\end{array}$ & $\begin{array}{ll}2 & 192 \\
2 & 189 \\
1 & 334 \\
1 & 341\end{array}$ & $\begin{array}{r}-27,7 \\
-\quad 4,0 \\
-28,7 \\
+\quad 17,4\end{array}$ & $\begin{array}{l}621 \\
577 \\
413 \\
361\end{array}$ \\
\hline 1972 & & & & & & \\
\hline $\begin{array}{l}\text { Dw Nana } \\
\text { Dw nana } \\
\text { dw Nana } \\
\text { dw nana }\end{array}$ & 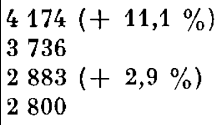 & $\begin{array}{l}+145(+7,9 \%) \\
-168 \\
+\quad 61(+4,5 \%) \\
-\quad 68\end{array}$ & $\begin{array}{l}+329(+7,2 \%) \\
+\quad 44 \\
+161(+3,9 \%) \\
+\quad 51\end{array}$ & $\begin{array}{l}2482 \\
2464 \\
1647 \\
1639\end{array}$ & $\begin{array}{l}+79,2 \\
+71,2 \\
+35,3 \\
+40,2\end{array}$ & $\begin{array}{r}1110 \\
1011 \\
799 \\
850\end{array}$ \\
\hline 1973 & & & & & & \\
\hline $\begin{array}{l}\text { Dw Nana } \\
\text { Dw nana } \\
\text { dw Nana } \\
\text { dw nana }\end{array}$ & 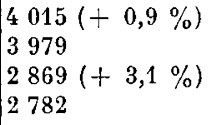 & $\begin{array}{l}+\quad 77(+3,3 \%) \\
-\quad 51 \\
+\quad 24(+1,3 \%) \\
-\quad 13\end{array}$ & $\begin{array}{l}+314(+3,3 \%) \\
+182 \\
+192(+3,5 \%) \\
+\quad 92\end{array}$ & $\begin{array}{ll}2 & 408 \\
2 & 482 \\
1 & 600 \\
1 & 577\end{array}$ & $\begin{array}{l}+63,1 \\
+65,6 \\
+41,0 \\
+\quad 25,5\end{array}$ & $\begin{array}{r}1125 \\
1145 \\
828 \\
866\end{array}$ \\
\hline
\end{tabular}

TABLEAU 3

Valeurs moyennes des performances par génotype ( $\left.\delta^{\star} \sigma^{\star}\right)$

\begin{tabular}{|c|c|c|c|c|c|}
\hline $\begin{array}{l}\text { Année } \\
\text { et génotype }\end{array}$ & $\begin{array}{l}\text { Aliment consommé } \\
(\mathrm{g} / 28 \mathrm{j})\end{array}$ & $\begin{array}{l}\text { Consommation } \\
\text { corrigée }\end{array}$ & $\begin{array}{l}\text { Consommation } \\
\text { partiellement } \\
\text { corrigée }\end{array}$ & $\begin{array}{c}\text { Pds corporel } \\
(\mathrm{g})\end{array}$ & $\begin{array}{c}\text { Variation } \\
\text { de poids } \\
(\mathrm{g} / 28 \mathrm{j})\end{array}$ \\
\hline $\begin{array}{c}1972 \\
\text { Dwdw Nana } \\
\text { Dwdw nana } \\
\text { dwdw Nana } \\
\text { dwdw nana }\end{array}$ & $\begin{array}{l}4295(+15,0 \%) \\
3694 \\
2648(+14,4 \%) \\
2291\end{array}$ & $\begin{array}{l}+323(+15,5 \%) \\
-296 \\
+156(+12,2 \%) \\
-145\end{array}$ & $\begin{array}{l}+464(+16,1 \%) \\
-178 \\
+\quad 304(+14,3 \%) \\
-\quad 48\end{array}$ & $\begin{array}{l}2699 \\
2806 \\
1791 \\
1790\end{array}$ & $\begin{array}{l}+31,7 \\
+\quad 26,7 \\
+\quad 55,3 \\
+\quad 36,0\end{array}$ \\
\hline $\begin{array}{c}1973 \\
\text { Dwdw Nana } \\
\text { Dwdw nana } \\
\text { dwdw Nana } \\
\text { dwdw nana }\end{array}$ & $\begin{array}{l}4583(+15,9 \%) \\
3908 \\
2763(+13,1 \%) \\
2424\end{array}$ & $\begin{array}{l}+339(+15,2 \%) \\
-311 \\
+186(+13,1 \%) \\
-228\end{array}$ & $\begin{array}{l}+480(+15,4 \%) \\
-180 \\
+408(+14,5 \%) \\
+\quad 40\end{array}$ & $\begin{array}{ll}3 & 125 \\
3 & 103 \\
1 & 798 \\
1 & 849\end{array}$ & $\begin{array}{l}+73,1 \\
+\quad 67,7 \\
+44,6 \\
+50,5\end{array}$ \\
\hline
\end{tabular}


l'un et l'autre sexe, et tant chez les nains que chez les normaux, n'ont pas été trouvés significativement différents en fonction du génotype $N a$ na ou na na (1). Ces coefficients ne diffèrent pas non plus selon le génotype pour $D w$. Le tableau 4 présente leur valeur, par année et par sexe, chez les oiseaux normaux et nains, ainsi que les coefficients de corrélation multiple correspondants.

\section{TABLEAU 4}

Equations de régression multiple de la consommation d'aliment sur le poids, la variation de poids et la production d'oufs

(g/périodes de 28 jours)

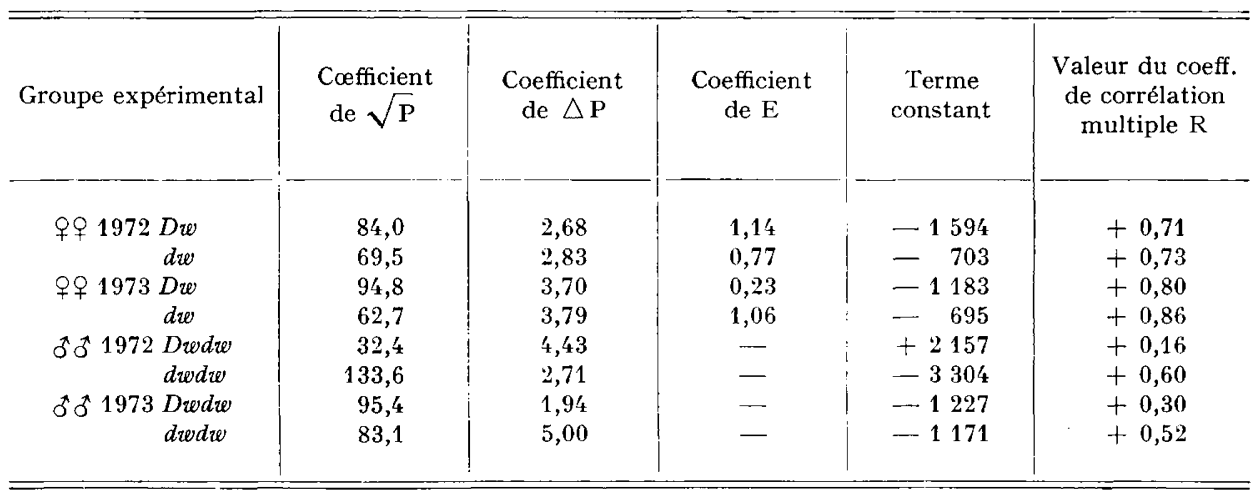

Concernant les variables dont les moyennes figurent aux tableaux 3 et 2 , les analyses de variance suivant les méthodes indiquées plus haut sont présentées dans les tableaux suivants (tabl. 5 et 6 ). Elles confirment le caractère significatif de l'effet du génotype au locus $N a$ sur la consommation corrigée totalement ou non, chez les poules $D w$ et $d w$, la différence de consommation observée n'atteignant pas le seuil 5 p. Ioo de signification. D'autre part, il est clair que le gène " cou nu " n'a pas ici d'effet significatif sur le poids corporel, la variation de poids et le poids des œufs pondus. L'effet " année " est faible et non significatif partout ; quant à l'effet " famille " il se trouve davantage accentué, dans l'ensemble, chez les poules $d w$. Enfin une interaction génotype pour $N a \times$ famille apparaît seulement dans un cas, comme il a été signalé plus haut.

Concernant les coqs, l'analyse de variance reflète clairement l'effet du gène " cou nu " sur la consommation d'aliment, corrigée ou non pour le poids et le gain de poids, et son absence d'effet sur ces deux dernières variables. L'effet " année " n'est significatif que pour le poids et la variation de celui-ci pour le génotype $D w d w$; l'effet " famille " est assez rarement significatif, mais par contre des interactions entre génotype au locus $\mathrm{Na}$ et famille apparaissent pour les variables qui traduisent la consommation alimentaire.

Finalement le tableau 7 présente l'unique analyse de variance (sur la consom-

(1) Il s'agit peut-être seulement d'un manque de sensibilité du test dû aux effectifs limités, car on peut penser qu'un effet du gène $N a$ sur la thermolyse devrait affecter le coefficient de $\sqrt{\mathrm{P}}$. 
CONSOMMA'TION ALIMEN'TAIRE E'T EMPLUMEMENT

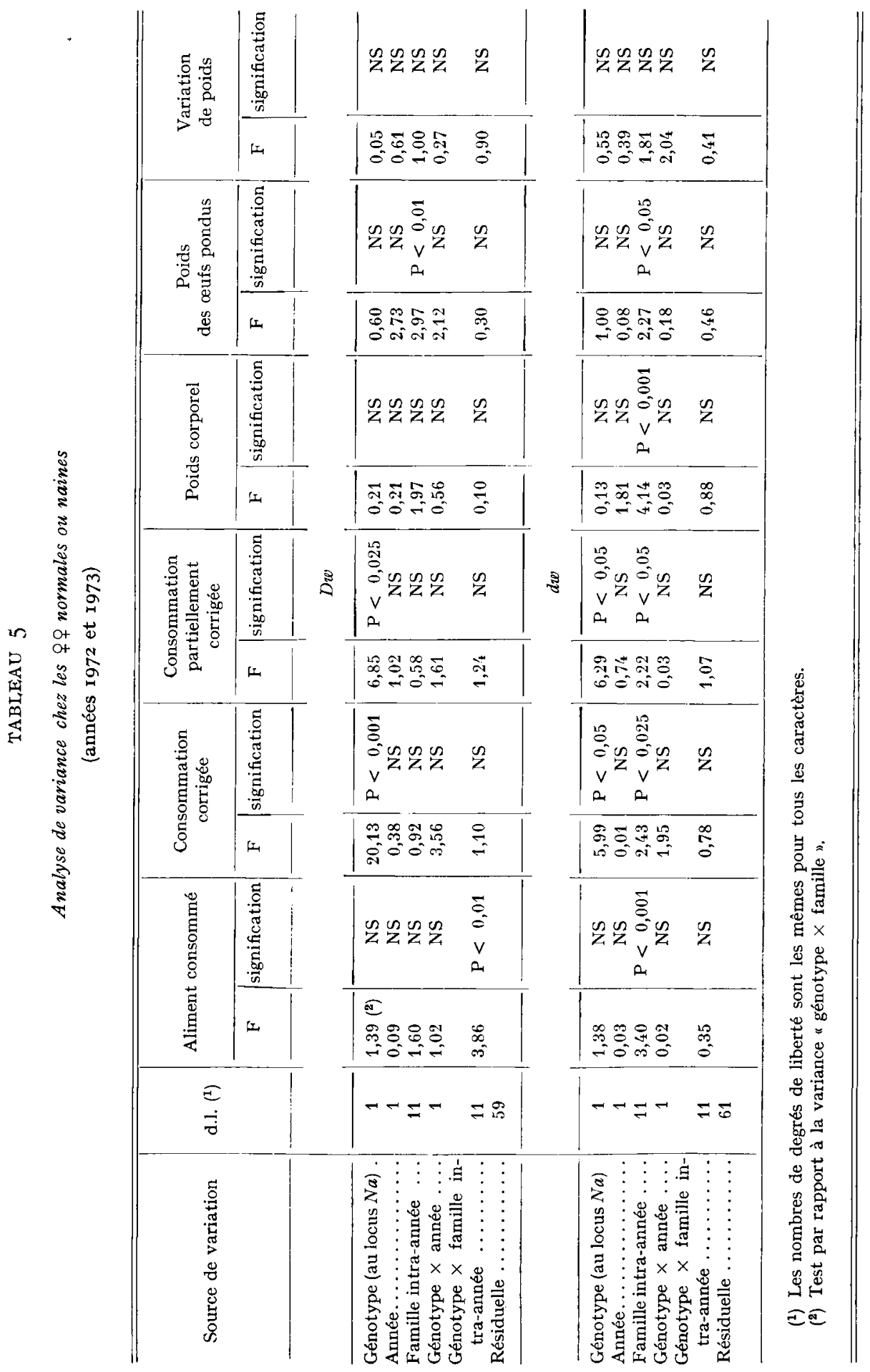




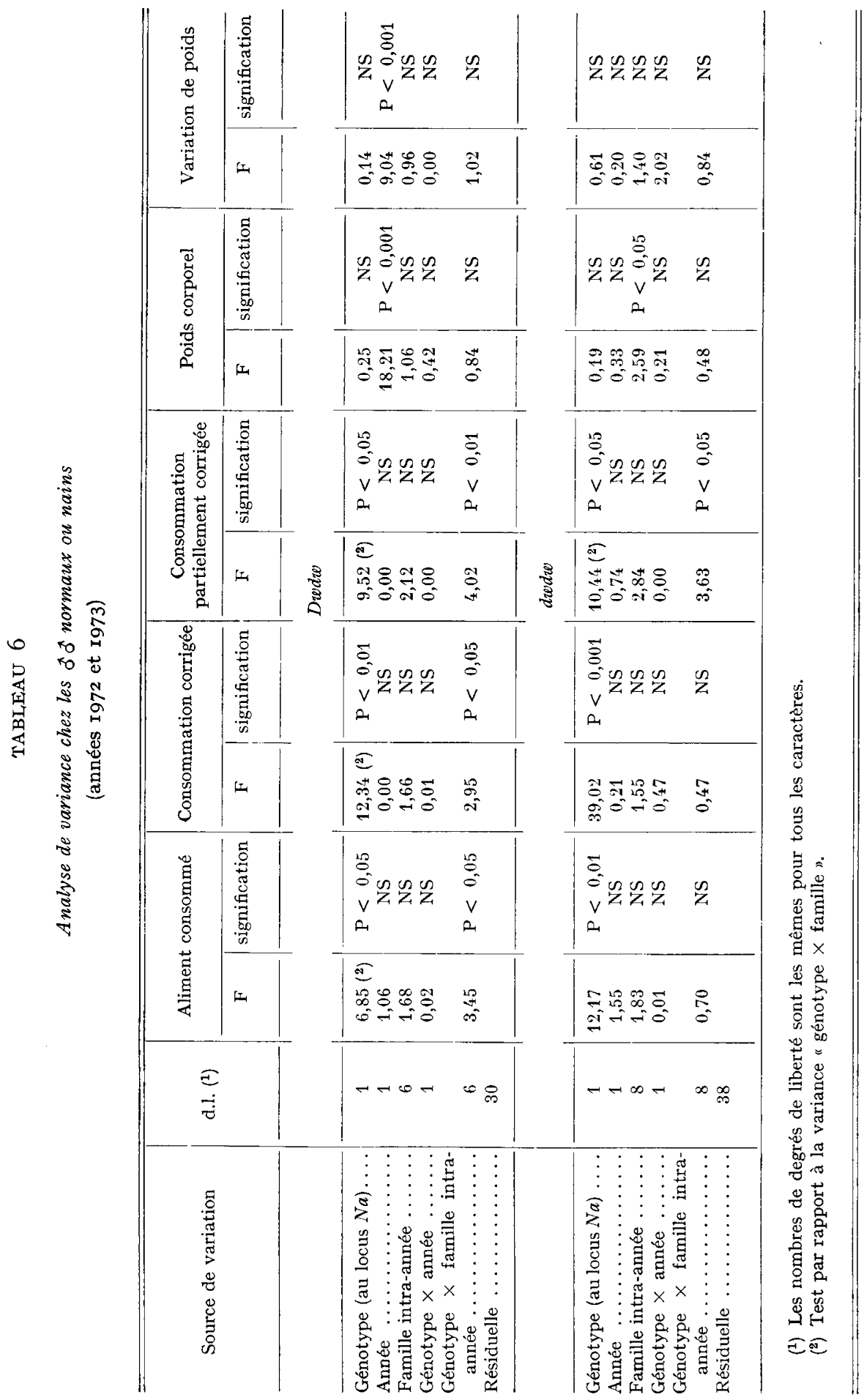


mation observée) combinant les facteurs contrôlés " génotype au locus $D w$ " et " génotype au locus $N a$ ». On y remarque l'absence de toute interaction significative, en particulier entre $D w$ et $N a$ chez les $q$ 우, malgré l'effet de $N a$ a première vue plus faible chez les naines.

\section{TABLEAU 7}

Analyse de variance de la quantité d'aliment consommée incluant l'effet du gène $\mathrm{dw}$

\begin{tabular}{|c|c|c|c|c|c|c|}
\hline \multirow{2}{*}{ Source de variation } & \multicolumn{3}{|c|}{ 우 } & \multicolumn{3}{|c|}{ పేర } \\
\hline & d.l. & $\mathrm{F}^{i}$ & signification & d.l. & $\mathrm{F}$ & signification \\
\hline $\begin{array}{l}\text { Génotype au locus } D w \ldots \ldots \\
\text { Génotype au locus } N a \ldots \ldots \ldots \\
\text { Année } \ldots \ldots \ldots \ldots \\
\text { Génotype } D w \times \text { année } \ldots \ldots \\
\text { Génotype } N a \times \text { année } \ldots \ldots \ldots \\
\text { Génotype } D w \times \text { génotype } N a \\
\text { Génotype } D w \times \text { génotype } N a \\
\quad \times \text { année } \ldots \ldots \ldots \ldots \ldots \ldots \\
\text { Résiduelle }(1) \ldots \ldots \ldots \ldots \ldots\end{array}$ & $\begin{array}{r}1 \\
1 \\
1 \\
1 \\
1 \\
1 \\
1 \\
1 \\
22\end{array}$ & $\begin{array}{r}146,60 \\
3,32 \\
0,30 \\
0,00 \\
0,83 \\
0,18 \\
\\
1,44\end{array}$ & $\begin{array}{c}\mathrm{P}<0,001 \\
\mathrm{NS}(\mathrm{P}<0,10) \\
\mathrm{NS} \\
\mathrm{NS} \\
\mathrm{NS} \\
\mathrm{NS} \\
\mathrm{NS}\end{array}$ & $\begin{array}{r}1 \\
1 \\
1 \\
1 \\
1 \\
1 \\
1 \\
1 \\
14\end{array}$ & $\begin{array}{r}186,40 \\
17,96 \\
2,60 \\
0,30 \\
0,01 \\
1,55\end{array}$ & $\begin{array}{c}\mathrm{P}<0,001 \\
\mathrm{P}<0,001 \\
\mathrm{NS} \\
\mathrm{NS} \\
\mathrm{NS} \\
\mathrm{NS} \\
\mathrm{NS}\end{array}$ \\
\hline
\end{tabular}

${ }^{(1)}$ La variance "génotype au locus $\mathrm{Na} \times$ famille " intra année, significative au seuil 5 p. 100, a été prise dans les deux cas comme variance résiduelle.

\section{DISCUSSION ET CONCLUSIONS}

L'augmentation de consommation alimentaire des animaux à plumage de type " cou nu " par rapport à ceux à emplumement complet se retrouve dans les deux sexes, tant chez les individus nains que chez ceux de taille normale, et ceci indépendamment de la taille corporelle, du gain de poids et de la ponte, sur lesquels le gène " cou nu " n'a pas d'effet significatif.

Cette augmentation, en pourcentage de la consommation globale, est plus importante chez les mâles, plus du double de celle observée chez les poules. Ceci s'explique vraisemblablement par le fait qu'une partie des besoins énergétiques de ces dernières concerne la ponte et n'est pas modifiée par le gène $\mathrm{Na}$. Chez les poules, nous avons déjà noté qu'au total, dans l'analyse du tableau 7 , la variance interaction entre génotypes aux loci $D w$ et $N a$ n'était pas significative. Cependant, cette analyse n'inclut pas l'année I97I, où, de même qu'en I972, l'effet associé au gène $\mathrm{Na}$ est plus faible chez les poules naines que chez les normales : seule l'année I973 ne fait pas apparaître la même différence, de sorte qu'il paraît difficile de conclure définitivement sur ce point. Par contre, le fait que, dans plusieurs cas, la variance interaction entre génotype au locus $\mathrm{Na}$ et famille soit significative suggère que des facteurs génétiques modifiant l'intensité de l'effet du gène $N a$ sur la consommation d'aliment sont présents dans la population étudiée. 
En conclusion, dans nos conditions tempérées d'environnement, la présence du gène " cou nu ", quoiqu'elle n'abaisse pas directement les performances, est nettement défavorable au rendement des pondeuses de taille normale, en produisant une surconsommation d'aliment sans élévation corrélative ni du poids, ni ces performances de ponte. Il fait peu de doute que ceci est dû̀ à l'accroissement des déperditions caloriques associé à un plumage réduit, et que l'effet serait accentué à température plus basse. Il en est de même des coqs adultes gardés pour la reproduction. Chez les poules naines, cet effet défavorable pourrait être compensé par l'influence positive sur le poids moyen des œufs et peut-être sur l'intensité de la ponte que nous avons mentionné en introduction (MÉRAT, données non publiées), mais il est douteux que le bilan total soit nettement avantageux dans nos conditions. Par contre, il est souhaitable de vérifier si, à température plus élevée, il peut exister un avantage corrélatif aux déperditions énergétiques accrues associées au caractère " cou nu ", en particulier chez des pondeuses naines. Un premier résultat dans ce sens sera publié ultérieurement.

Reçu pour publication en février 1974.

\section{REMERCIEMENTS}

Nous remercions M. J. Guillaume, Station de Recherches avicoles, I.N.R.A., Nouzill.y, et M. F. H. Ricard, Station Expérimentale d'Aviculture du Magneraud, I.N.R.A, de leurs critiques et suggestions constructives concernant ce manuscrit.

\section{SUMMARY}

INDIVIDUAL FEED CONSUMPTION OF "NAKED NECK ) ( $N a$ GENE) OR NORMAL HENS IN PRESENCE OR ABSENCE OF THE DWARF GENE

Individual feed consumption, observed or corrected for body weight, weight gain and egg production, was compared for "naked neck " $(N a n a)$ or $n a n x$ hens, in presence or absence of the dwarf gene $d w$ : The excess of " corrected " consumption of "naked neck" hens in our temperature conditions (about $18-20^{\circ} \mathrm{C}$ on the average) is significant. In males, the difference, in percent, is more considerable than in females. It is of the same order in dwarfs as in normal-sized cocks, while in hens, our data suggest that it may be lower in presence of the $d w$ gene.

\section{RÉFÉRENCES BIBLIOGRAPHIQUES}

Bernier P. E., Arscott G. H., I96o. Relative efficiency of sex-linked dwarf layers and their normal sisters. Poult. Sci., 39, I234-I235.

Byerly T. C., I94I. Feeds and other costs of producing market eggs. Univ. Maryland Agric. Exp. Sta. Bull., $\mathrm{n}^{0}$ Ar.

Hutr F. B., 1949. Genetics of the fowl. McGraw Hill, New-York.

Hurt F. B., I953. Sex-linked dwarfism in the fowl. Genetics, 38, 670.

Hutr F. B., I959. Sex-linked dwarfism in the fowl. J. Hered. 50, 209-221.

Kendall M. G., Stuart A., I967. The advanced theory of statistics. Griffin, London. 
Mérat P., Ricard F. H., I974. Étude d'un gène de nanisme lié au sexe chez la poule : importance des lipides corporels et gain de poids chez la pondeuse. Ann. Génét. Sél. Anim., (sous presse).

Prod'номмe J., I965. Calcul d'une équation permettant de prévoir la consommation alimentaire de pondeuses en fonction de leurs performances. Ann. Zooteeh., 14, 335-339.

Simon J., I972. Influence du gène de nanisme $(d w)$, du cou nu $(N a)$ et du rythme d'alimentation sur la croissance et le comportement alimentaire du poulet. Ann. Génét. Sél. anim., 4, 305-3io.

Snedecor G. W., Cochran W. G., I967. Statistical methods. Iowa State University Press, Ames.

Touchburn S. P., Blum J. C., 1972. Effects of the genes for dwarfism $(d w)$ and naked neck $(N a)$ on chick growth and lipid metabolism. Ann. Génét. Sél. anim., 4, 3I I-3I6. 